\title{
CONNECTIVITY FOR BRIDGE-ADDABLE MONOTONE GRAPH CLASSES
}

\author{
LOUIGI ADDARIO-BERRY, COLIN MCDIARMID, AND BRUCE REED
}

\begin{abstract}
A class $\mathcal{A}$ of labelled graphs is bridge-addable if if for all graphs $G$ in $\mathcal{A}$ and all vertices $u$ and $v$ in distinct connected components of $G$, the graph obtained by adding an edge between $u$ and $v$ is also in $\mathcal{A}$; the class $\mathcal{A}$ is monotone if for all $G \in \mathcal{A}$ and all subgraphs $H$ of $G$, we have $H \in \mathcal{A}$. We show that for any bridge-addable, monotone class $\mathcal{A}$ whose elements have vertex set $\{1, \ldots, n\}$, the probability that a uniformly random element of $\mathcal{A}$ is connected is at least $\left(1-o_{n}(1)\right) e^{-\frac{1}{2}}$, where $o_{n}(1) \rightarrow 0$ as $n \rightarrow \infty$. This establishes the special case of the conjecture of [17] when the condition of monotonicity is added. This result has also been obtained independently by Kang and Panagiotiou (2011).
\end{abstract}

\section{INTRODUCTION}

Given a class $\mathcal{A}$ of graphs, we say that $\mathcal{A}$ is bridge-addable (or weakly addable) if for all graphs $G$ in $\mathcal{A}$ and all vertices $u$ and $v$ in distinct connected components of $G$, the graph obtained by adding an edge between $u$ and $v$ is also in $\mathcal{A}$. The concept of bridge-addability was introduced in McDiarmid, Steger and Welsh [16] in the course of studying random planar graphs. It was shown there that, for a uniformly random element of a finite nonempty bridge-addable class $\mathcal{A}$ of labelled graphs, the probability that it is connected is at least $e^{-1}$.

As well as the class of planar graphs, other examples of bridge-addable graph classes include forests, graphs with tree-width at most $k$, graphs embeddable on any fixed surface, and more generally any minor-closed graph class with cut-point-free excluded minors; triangle-free graphs, and more generally $H$-free graphs for any two-edge-connected graph $H$; and $k$-colourable graphs.

It is well-known that there are $n^{n-2}$ trees on $n$ labelled vertices, Cayley [8]. Together with a result of Rényi [19] (see also Moon [18]) that the corresponding number of forests is asymptotic to $e^{\frac{1}{2}} n^{n-2}$, we see that, for a uniformly random forest on $n$ labelled vertices, the probability that it is connected is asymptotically $e^{-\frac{1}{2}}$.

In McDiarmid, Steger and Welsh [17] it was suggested that if connectivity is desired then the worst possible example of a bridge-addable graph class is the class of forests. More precisely, they conjectured that the lower bound of $e^{-1}$ on the probability of connectedness for a bridge-addable graph class can be improved asymptotically to $(1+o(1)) e^{-\frac{1}{2}}$. (They assumed also that the graph class was closed under isomorphism.) Recently, Balister, Bollobás and Gerke [1, 2] took a first step towards proving this conjecture, proving an asymptotic lower bound of $e^{-0.7983}$.

Observe that the examples above of bridge-addable graph classes, and many other interesting examples of such graph classes, also satisfy the property of being monotone (decreasing); that is, given a graph $G$ in $\mathcal{A}$, each graph obtained by deleting edges from $G$ is also in $\mathcal{A}$. In this paper we investigate the probability of connectivity of a uniformly random element of a monotone bridge-addable graph class; for such graph classes, we prove the conjectured lower bound. Our method relies upon a reduction to weighted random forests; and in particular to the properties of weighted random trees. 
For several bridge-addable graph classes, including some of those mentioned above, the asymptotic probability of connectedness has recently been determined - see the last section below.

Let us now state our theorem. A bridge in a graph $G$ is an edge $e$ such that $G-e$ has strictly more connected components than $G$. We say that a class $\mathcal{A}$ of graphs is bridgealterable if for any graph $G$ and bridge $e$ in $G, G$ is in $\mathcal{A}$ if and only if $G-e$ is. We remark that if $\mathcal{A}$ is bridge-alterable then it is bridge-addable, and if it is both bridge-addable and monotone then it is bridge-alterable.

Theorem 1. For any $\varepsilon>0$ there exists $W_{0}$ such that, if $W \geq W_{0}$ and $\mathcal{A}$ is a non-empty bridge-alterable class of graphs on $\{1, \ldots, W\}$, and if $\mathbf{G}$ is a uniformly random element of $\mathcal{A}$, then

$$
\mathbf{P}\{\mathbf{G} \text { is connected }\} \geq(1-\varepsilon) e^{-\frac{1}{2}} .
$$

This result was announced independently by Kang and Panagiotou while the present paper was under (slow) revision following referee reports, with at that time a weaker form of the above theorem. Their proof in [15] starts like our proof here but then proceeds very differently, with a clever induction involving merging vertices when counting forests with two components (where the vertices correspond to bridgeless graphs).

In the next section we describe a reduction which allows us to establish Theorem 10 by proving that, for a random forest with an appropriately chosen distribution, full connectivity (the event that the forest is a tree) is almost twice as likely as the event that the forest has precisely two connected components (the precise statement appears as Lemma 5 , below). In the following two sections we explain how Lemma 5 will yield Theorem 1 , and then prove Lemma 5 . Finally we make some concluding remarks.

\section{A Reduction to Weighted Forests}

In this section we assume that the conditions of Theorem 1 are satisfied. For any class $\mathcal{A}$ of graphs, let $\mathcal{A}_{n}$ denote the set of graphs in $\mathcal{A}$ on the vertex set $\{1, \ldots, n\}$. It is convenient to consider graphs on $W$ vertices. We shall prove Theorem 1 by partitioning $\mathcal{A}_{W}$ and showing that an inequality such as (11) holds for a uniformly random element of each block of the partition. (This step of our proof is essentially Lemma 2.1 of [1].)

Definition 2. Given a graph $G$, let $b(G)$ be the graph obtained by removing all bridges from $G$. We say $G$ and $G^{\prime}$ are equivalent if $b(G)=b\left(G^{\prime}\right)$, and in this case write $G \sim G^{\prime}$. For a graph $G$, let $[G]$ be the set of graphs $G^{\prime}$ for which $b\left(G^{\prime}\right)=b(G)$.

It is easily seen that $\sim$ is an equivalence relation on graphs, and thus we always have $[G]=[b(G)]$. Furthermore, if $G \in \mathcal{A}_{W}$ then as $\mathcal{A}$ is closed under deleting bridges, $b(G) \in$ $\mathcal{A}_{W}$, and as $\mathcal{A}$ is bridge-addable, we have $[G] \subseteq \mathcal{A}_{W}$. It follows that $\mathcal{A}_{W}$ can be written as a union of some set of disjoint equivalence classes $\left[G_{1}\right],\left[G_{2}\right], \ldots$ To prove Theorem 1 , we will in fact prove:

Claim 3. For any graph $G \in \mathcal{A}_{W}$, if $H$ is a uniformly random element of $[G]$ then

$$
\mathbf{P}\{H \text { is connected }\} \geq e^{-\frac{1}{2}+o(1)},
$$

where $o(1) \rightarrow 0$ as $W \rightarrow \infty$.

Clearly, Theorem 1 immediately follows from Claim 3, and it thus remains to prove Claim 3. Fix a bridgeless graph $G$ on vertex set $\{1, \ldots, W\}$ and let $\mathcal{B}=[G]$. Write $C_{1}, \ldots, C_{n}$ for the components of $G$, and let $w_{i}=\left|V\left(C_{i}\right)\right|$ for $i=1, \ldots, n$, so $W=\sum_{i=1}^{n} w_{i}$. We remark that since the components $C_{1}, \ldots, C_{n}$ are bridgeless, either $w_{i}=1$ or $w_{i} \geq 3$ for all $i \in\{1, \ldots, n\}$ (though we shall not use this fact). We denote by $\vec{w}$ the vector 
$\left(w_{1}, \ldots, w_{n}\right)$. We use $\vec{w}$ to define a probability measure on the set $\mathcal{F}_{n}$ of forests with vertex set $\{1, \ldots, n\}$. Given $F \in \mathcal{F}_{n}$, let

$$
\operatorname{mass}(F)=\operatorname{mass}_{\vec{w}}(F)=\prod_{i=1}^{n} w_{i}^{d_{F}(i)},
$$

where $d_{F}(i)$ denotes the degree of vertex $i$ in the forest $F$. Also, let $K=\sum_{F \in \mathcal{F}_{n}} \operatorname{mass}(F)$, and let $\mathbf{F}$ be a random element of $\mathcal{F}_{n}$ with $\mathbf{P}\{\mathbf{F}=F\}=\operatorname{mass}(F) / K$ for all $F \in \mathcal{F}_{n}$. We say $\mathbf{F}$ is distributed according to $\vec{w}$; when we wish to highlight the distribution of $\mathbf{F}$, we will sometimes write $\mathbf{F}_{\vec{w}}$ in place of $\mathbf{F}$. For our purposes, the key fact about such a random forest is the following:

Lemma 4. For a uniformly random element $\mathbf{H}$ of $\mathcal{B}$,

$$
\mathbf{P}\{\mathbf{H} \text { is connected }\}=\mathbf{P}\{\mathbf{F} \text { is connected }\} .
$$

Proof. We construct a flow from $\mathcal{B}$ to $\mathcal{F}_{n}$ in the following fashion: given $G \in \mathcal{B}$, let $f(G)$ be the graph obtained from $G$ by contracting $C_{i}$ to a single point for each $i=1, \ldots, n$; then $f(G) \in \mathcal{F}_{n}$, and for each $F \in \mathcal{F}_{n}$, the set $f^{-1}(F)$ has cardinality precisely $\prod_{i=1}^{n} w_{i}^{d_{F}(i)}$. Since $G \in \mathcal{B}$ in connected if and only if $f(G)$ is connected, it follows that

$$
\begin{aligned}
\mathbf{P}\{\mathbf{H} \text { is connected }\} & =\frac{\mid\{G \in \mathcal{B}: G \text { is connected }\} \mid}{|\mathcal{B}|} \\
& =\frac{\sum_{\left\{F \in \mathcal{F}_{n}: F \text { is connected }\right\}}\left|f^{-1}(F)\right|}{\sum_{F \in \mathcal{F}_{n}}\left|f^{-1}(F)\right|} \\
& =\frac{\sum_{\left\{F \in \mathcal{F}_{n}: F \text { is connected }\right\}} \operatorname{mass}(F)}{K} \\
& =\mathbf{P}\{\mathbf{F} \text { is connected }\}
\end{aligned}
$$

as required.

To prove Claim [3, it therefore suffices to show that for such a random forest $\mathbf{F}$, $\mathbf{P}\{\mathbf{F}$ is connected $\} \geq e^{-\frac{1}{2}+o(1)}$, where $o(1)$ tends to zero as $W \rightarrow \infty$.

For $i=1, \ldots, n$, let $\mathcal{F}_{n, i}$ be the set of elements of $\mathcal{F}_{n}$ with $i$ components (so $F \in \mathcal{F}_{n, 1}$ precisely if $F$ is connected). For larger $i$ set $\mathcal{F}_{n, i}=\emptyset$. It turns out that bounds on $\mathbf{P}\{\mathbf{F}$ is connected $\}$ follow from bounds on the ratio between $\mathbf{P}\left\{F \in \mathcal{F}_{n, 2}\right\}$ and $\mathbf{P}\left\{F \in \mathcal{F}_{n, 1}\right\}$. More precisely, Claim 3 follows from Lemma 4 and the following lemma.

Lemma 5. For all $\varepsilon>0$, for $W$ sufficiently large, for all $\vec{w}=\left(w_{1}, \ldots, w_{n}\right)$ with $\sum_{j=1}^{n} w_{j}=W$,

$$
\mathbf{P}\left\{\mathbf{F} \in \mathcal{F}_{n, 2}\right\} \leq(1+\varepsilon) \frac{1}{2} \mathbf{P}\left\{\mathbf{F} \in \mathcal{F}_{n, 1}\right\} .
$$

In Section 3 we explain how to use Lemma 5 to prove Claim 3 in Section 4 we prove Lemma 5 .

\section{Proof of Claim 3 assuming Lemma 5}

The proof of Claim 3 proceeds somewhat differently depending on the value of the ratio of $n$ and $W$. When $W$ is much larger than $n$, the proof is rather straightforward, and in fact does not require Lemma 5 at all, but rather Lemma 7 below. In both cases, however, we compare the probability masses of $\mathcal{F}_{n, i}$ and of $\mathcal{F}_{n, i+1}$ by double-counting edge-weights in a bipartite graph.

Given a graph $G$, let $c(G)$ be the set of connected components of $G$. Given a forest $F \in \mathcal{F}_{n}$ and $T \in c(F)$, let $w(T)=\sum_{i \in V(T)} w_{i}$. Consider forests $F, F^{\prime} \in \mathcal{F}_{n}$ such that $F^{\prime}$ 
can be obtained from $F$ by the addition of an edge $e$. Writing $T \neq T^{\prime} \in c(F)$ as shorthand for $\left\{\left\{T, T^{\prime}\right\} \subseteq c(F): T \neq T^{\prime}\right\}$, we let

$$
\varphi\left(F^{\prime}, F\right)=\frac{\operatorname{mass}\left(F^{\prime}\right)}{\sum_{T \neq T^{\prime} \in c(F)} w(T) w\left(T^{\prime}\right)} .
$$

For all other pairs $F, F^{\prime}$, we let $\varphi\left(F^{\prime}, F\right)=0$. We use $\varphi$ in a standard double-counting argument. We will use the next preliminary lemma also in Section 4.

Lemma 6. For all positive integers $W$ and all positive integer weight vectors $\vec{w}=$ $\left(w_{1}, \ldots, w_{n}\right)$ with $\sum_{j=1}^{n} w_{j}=W$, and for each $i=1, \ldots, n-1$

$$
\sum_{F^{\prime} \in \mathcal{F}_{n, i}} \sum_{F \in \mathcal{F}_{n, i+1}} \varphi\left(F^{\prime}, F\right)=\sum_{F \in \mathcal{F}_{n, i+1}} \operatorname{mass}(F)=K \cdot \mathbf{P}\left\{\mathbf{F} \in \mathcal{F}_{n, i+1}\right\} .
$$

Proof. Given $i \in\{1, \ldots, n-1\}$ and $F \in \mathcal{F}_{n, i+1}$, if $F^{\prime} \in \mathcal{F}_{n, i}$ is obtained from $F$ by the addition of edge $u v$, then $\operatorname{mass}\left(F^{\prime}\right)=\operatorname{mass}(F) \cdot w_{u} \cdot w_{v}$. We thus have

$$
\begin{aligned}
& \sum_{F^{\prime} \in \mathcal{F}_{n, i}} \varphi\left(F^{\prime}, F\right) \\
= & \left(\sum_{T \neq T^{\prime} \in c(F)} \sum_{u \in V(T), v \in V\left(T^{\prime}\right)} \operatorname{mass}(F) \cdot w_{u} \cdot w_{v}\right) \cdot\left(\frac{1}{\sum_{T \neq T^{\prime} \in c(F)} w(T) w\left(T^{\prime}\right)}\right) \\
= & \frac{\operatorname{mass}(F)}{\sum_{T \neq T^{\prime} \in c(F)} w(T) w\left(T^{\prime}\right)} \cdot\left(\sum_{T \neq T^{\prime} \in c(F)} \sum_{u \in V(T), v \in V\left(T^{\prime}\right)} w_{u} \cdot w_{v}\right)=\operatorname{mass}(F) .
\end{aligned}
$$

The equation (4) now follows on summing over $F$.

Lemma 7. For all positive integers $W$ and all positive integer weight vectors $\vec{w}=$ $\left(w_{1}, \ldots, w_{n}\right)$ with $\sum_{j=1}^{n} w_{j}=W$, and for each $i=1, \ldots, n-1$

$$
\mathbf{P}\left\{\mathbf{F} \in \mathcal{F}_{n, i+1}\right\} \leq \frac{\mathbf{P}\left\{\mathbf{F} \in \mathcal{F}_{n, i}\right\}(n / W)}{i},
$$

Proof. Fix $i$ with $1 \leq i \leq n-1$. By the definition of $\varphi$, for all $F^{\prime} \in \mathcal{F}_{n, i}$ we have

$$
\sum_{F \in \mathcal{F}_{n, i+1}} \varphi\left(F^{\prime}, F\right)=\operatorname{mass}\left(F^{\prime}\right) \cdot \sum_{e \in E\left(F^{\prime}\right)} \frac{1}{\sum_{T \neq T^{\prime} \in c\left(F^{\prime}-e\right)} w(T) w\left(T^{\prime}\right)} .
$$

It is well known that for any set of positive integers $a_{1}, \ldots, a_{i+1}$ with $\sum_{j=1}^{i+1} a_{j}=W$,

$$
\sum_{1 \leq j<k \leq i+1} a_{j} a_{k} \geq i(W-i)+\left(\begin{array}{l}
i \\
2
\end{array}\right)
$$

[To see this, if $a_{1} \geq a_{2} \geq 2$ then let $a_{1}^{\prime}=a_{1}+1, a_{2}^{\prime}=a_{2}-1$ and $a_{j}^{\prime}=a_{j}$ for each $j \geq 3$. Then with sums as above, $\sum_{j<k} a_{j}^{\prime} a_{k}^{\prime}-\sum_{j<k} a_{j} a_{k}=a_{1}^{\prime} a_{2}^{\prime}-a_{1} a_{2}=-a_{1}+a_{2}-1<0$. Hence the sum is minimised when there are $i$ entries 1 and one entry $W-i$.] It follows that, for any $F^{\prime} \in \mathcal{F}_{n, i}$ and any $e \in E\left(F^{\prime}\right)$, we have

$$
\sum_{T \neq T^{\prime} \in c\left(F^{\prime}-e\right)} w(T) w\left(T^{\prime}\right) \geq i(W-i)+\left(\begin{array}{l}
i \\
2
\end{array}\right) \geq i(W-i) .
$$

Since, if $F^{\prime} \in \mathcal{F}_{n, i}$ then $F^{\prime}$ has exactly $n-i$ edges, it follows from (6) and (7) that for all $F^{\prime} \in \mathcal{F}_{n, i}$

$$
\sum_{F \in \mathcal{F}_{n, i+1}} \varphi\left(F^{\prime}, F\right) \leq \operatorname{mass}\left(F^{\prime}\right) \cdot(n-i) \cdot \frac{1}{i(W-i)} \leq \operatorname{mass}\left(F^{\prime}\right) \cdot \frac{1}{i} \cdot \frac{n}{W},
$$


so

$$
\begin{aligned}
\sum_{F^{\prime} \in \mathcal{F}_{n, i}} \sum_{F \in \mathcal{F}_{n, i+1}} \varphi\left(F^{\prime}, F\right) & \leq \sum_{F^{\prime} \in \mathcal{F}_{n, i}} \operatorname{mass}\left(F^{\prime}\right) \cdot \frac{1}{i} \cdot \frac{n}{W} \\
& =K \cdot \mathbf{P}\left\{\mathbf{F} \in \mathcal{F}_{n, i}\right\} \cdot \frac{1}{i} \cdot \frac{n}{W},
\end{aligned}
$$

and (5) follows by combining this last result and Lemma 6 .

The case $n / W \leq \frac{1}{2}$ of Lemma 5 follows immediately from the above lemma; and indeed in this case we may complete the proof directly without using the next two lemmas see the last sentence of this section. To explain why Lemma 5 implies Claim 3 when $n$ is not much smaller than $W$, it turns out to be useful to prove a slightly more general implication.

For each finite non-empty set $V$ of positive integers, let $\mathcal{G}(V)$ be the set of all graphs on the vertex set $V$, and let $\mathcal{G}^{k}(V)$ be the set of all graphs in $\mathcal{G}(V)$ with exactly $k$ components. Also, write $\mathcal{G}_{n}$ for $\mathcal{G}(\{1, \ldots, n\})$, and $\mathcal{G}_{n}^{k}$ for $\mathcal{G}^{k}(\{1, \ldots, n\})$. For each positive integer $n$, let $\mu_{n}$ be a measure on the set of all graphs with vertex set a subset of $\{1, \ldots, n\}$, which is multiplicative on components (that is, if $G$ has components $H_{1}, \ldots, H_{k}$, then $\left.\mu_{n}(G)=\prod_{i=1}^{k} \mu_{n}\left(H_{i}\right)\right)$.

Lemma 8. Suppose there exist $x>0$ and integers $n \geq m_{0} \geq 1$ such that

$$
\mu_{n}\left(\mathcal{G}^{2}(V)\right) \leq x \mu_{n}\left(\mathcal{G}^{1}(V)\right) \quad \text { for all } V \subseteq\{1, \ldots, n\} \text { with }|V| \geq m_{0} .
$$

Let $k$ be a positive integer and suppose that $n \geq k m_{0}$. Then

$$
\mu_{n}\left(\mathcal{G}_{n}^{k+1}\right) \leq \frac{x}{k} \mu_{n}\left(\mathcal{G}_{n}^{k}\right) .
$$

Proof. Let $\mathcal{A}$ be the collection of all sets $\left\{H_{1}, \ldots, H_{k-1}\right\}$ of $k-1$ connected graphs such that the vertex sets $V\left(H_{i}\right)$ are pairwise disjoint subsets of $\{1, \ldots, n\}$ and

$$
\max _{1 \leq i \leq k-1}\left|V\left(H_{i}\right)\right|<n-\sum_{i=1}^{k-1}\left|V\left(H_{i}\right)\right| .
$$

Let $\mathcal{H}=\left\{H_{1}, \ldots, H_{k-1}\right\} \in \mathcal{A}$, let $V_{\mathcal{H}}=\{1, \ldots, n\} \backslash\left(\bigcup_{i=1}^{k-1} V\left(H_{i}\right)\right)$, and note that $\left|V_{\mathcal{H}}\right|>$ $\max _{1 \leq i \leq k-1}\left|V\left(H_{i}\right)\right|$ and $\left|V_{\mathcal{H}}\right| \geq m_{0}$. For $j=k$ and $k+1$, let $\mathcal{G}_{n}^{j}(\mathcal{H})$ denote the set of all graphs $G$ in $\mathcal{G}_{n}^{j}$ such that $H_{1}, \ldots, H_{k-1}$ are each components of $G$. Then, letting $\alpha=\prod_{i=1}^{k-1} \mu_{n}\left(H_{i}\right)$, by the multiplicativity of $\mu_{n}$ and by (8) we have

$$
\mu_{n}\left(\mathcal{G}_{n}^{k+1}(\mathcal{H})\right)=\alpha \cdot \mu_{n}\left(\mathcal{G}^{2}\left(V_{\mathcal{H}}\right)\right) \leq x \alpha \cdot \mu_{n}\left(\mathcal{G}^{1}\left(V_{\mathcal{H}}\right)\right)=x \cdot \mu_{n}\left(\mathcal{G}_{n}^{k}(\mathcal{H})\right) .
$$

Next, consider any graph $G \in \mathcal{G}_{n}^{k+1}$, and suppose that $G$ has components $G_{1}, \ldots, G_{k+1}$, where $\left|V\left(G_{1}\right)\right| \leq \ldots \leq\left|V\left(G_{k+1}\right)\right|$. For each set $\mathcal{H}$ formed by picking any $k-1$ of the graphs $G_{1}, \ldots, \bar{G}_{k}$, we have $\mathcal{H} \in \mathcal{A}$ and $G \in \mathcal{G}_{n}^{k+1}(\mathcal{H})$. It follows that

$$
k \cdot \mu_{n}\left(\mathcal{G}_{n}^{k+1}\right) \leq \sum_{\mathcal{H} \in \mathcal{A}} \mu_{n}\left(\mathcal{G}_{n}^{k+1}(\mathcal{H})\right) .
$$

Applying (10) to bound the right-hand side of (11), we obtain

$$
k \cdot \mu_{n}\left(\mathcal{G}_{n}^{k+1}\right) \leq x \cdot \sum_{\mathcal{H} \in \mathcal{A}} \mu_{n}\left(\mathcal{G}_{n}^{k}(\mathcal{H})\right) .
$$

Furthermore, the sets $\left\{\mathcal{G}_{n}^{k}(\mathcal{H}): \mathcal{H} \in \mathcal{A}\right\}$ are pairwise disjoint subsets of $\mathcal{G}_{n}^{k}$, so $\sum_{\mathcal{H} \in \mathcal{A}} \mu_{n}\left(\mathcal{G}_{n}^{k}(\mathcal{H})\right) \leq$ $\mu_{n}\left(\mathcal{G}_{n}^{k}\right)$, which combined with (12) yields that

$$
k \cdot \mu_{n}\left(\mathcal{G}_{n}^{k+1}\right) \leq x \cdot \mu_{n}\left(\mathcal{G}_{n}^{k}\right),
$$

which completes the proof. 
Lemma 8 allows us to derive bounds on the ratio between $\mathbf{P}\left\{\mathbf{F} \in \mathcal{F}_{n, i+1}\right\}$ and $\mathbf{P}\left\{\mathbf{F} \in \mathcal{F}_{n, i}\right\}$ for $i>1$.

Lemma 9. Suppose that there exist $0<\gamma<1$ and $m_{0}>0$, such that for any positive integer weights $\vec{w}=\left(w_{1}, \ldots, w_{n}\right)$ with $\sum_{k=1}^{n} w_{k} \geq m_{0}, \mathbf{P}\left\{\mathbf{F}_{\vec{w}} \in \mathcal{F}_{n, 2}\right\} \leq \gamma \mathbf{P}\left\{\mathbf{F}_{\vec{w}} \in \mathcal{F}_{n, 1}\right\}$. Fix any positive integer $j$. Then for $W$ sufficiently large, for all integers $i$ with $1 \leq i \leq j$ and any positive integer weights $\vec{w}=\left\{w_{1}, \ldots, w_{n}\right\}$ with $\sum_{k=1}^{n} w_{k}=W$,

$$
\mathbf{P}\left\{\mathbf{F} \in \mathcal{F}_{n, i+1}\right\} \leq \frac{\gamma \mathbf{P}\left\{\mathbf{F} \in \mathcal{F}_{n, i}\right\}}{i} .
$$

Proof. Suppose $\gamma$ and $m_{0}$ satisfy the hypotheses of the lemma, and fix some positive integer $j$. Observe first that, by Lemma 7, the inequality (13) holds if $n \leq \gamma W$. We may thus assume that $n>\gamma W$.

Let $n \geq m_{0}$ and consider any weights $w_{1}, \ldots, w_{n}$. Define $\mu_{n}(G)$ for each graph $G$ with vertex set $V \subseteq\{1, \ldots, n\}$ by setting $\mu_{n}(G)=\prod_{i \in V} w_{i}^{d_{G}(i)}$ if $G$ is a forest and $\mu_{n}(G)=0$ otherwise. Then $\mu_{n}$ is multiplicative on components, and by the hypotheses of the lemma, for each $V \subseteq\{1, \ldots, n\}$ with $\sum_{i \in V} w_{i} \geq m_{0}$ we have

$$
\mu_{n}\left(\mathcal{G}^{2}(V)\right) \leq \gamma \mu_{n}\left(\mathcal{G}^{1}(V)\right)
$$

Now we may use Lemma 8 to obtain

$$
\mu_{n}\left(\mathcal{G}_{n}^{k+1}\right) \leq \frac{\gamma}{k} \mu_{n}\left(\mathcal{G}_{n}^{k}\right)
$$

whenever $n \geq k m_{0}$. Since $n \geq k m_{0}$ whenever $W \geq k m_{0} / \gamma$, Lemma 9 follows.

Proof of Claim 3 assuming Lemma囵. Fix $\alpha$ with $0<\alpha<1$, and choose $j$ large enough that $2 / j ! \leq \alpha / 2$. Let $\varepsilon>0$ be small enough that $(1-\alpha / 2) /(1+\varepsilon)^{j} \geq 1-\alpha$. We apply Lemma 9 with $\gamma=(1+\varepsilon) \frac{1}{2}$ (Lemma 5 guarantees that there exists $m_{0}>0$ such that the hypotheses of Lemma 9 hold with this choice of $m_{0}$ and $\gamma$ ). It follows that for $W$ large enough, for all $i$ with $1 \leq i \leq j$ we have

$$
\mathbf{P}\left\{\mathbf{F} \in \mathcal{F}_{n, i+1}\right\} \leq(1+\varepsilon)^{i} \frac{\mathbf{P}\left\{\mathbf{F} \in \mathcal{F}_{n, 1}\right\}}{2^{i} i !} .
$$

Furthermore, writing $\kappa(F)$ for the number of connected components of $F$,

$$
\begin{aligned}
1 & =\sum_{i=0}^{n-1} \mathbf{P}\left\{\mathbf{F} \in \mathcal{F}_{n, i+1}\right\} \\
& \leq(1+\varepsilon)^{j} \sum_{i=0}^{j-1} \frac{\mathbf{P}\left\{\mathbf{F} \in \mathcal{F}_{n, 1}\right\}}{2^{i} i !}+\mathbf{P}\{\kappa(\mathbf{F}) \geq j+1\} .
\end{aligned}
$$

By Lemma 7, for all $i \geq 1$,

$$
\mathbf{P}\left\{\mathbf{F} \in \mathcal{F}_{n, i+1}\right\} \leq \frac{(n / W)^{i}}{i !} \leq \frac{1}{i !},
$$

from which it follows that for all $k \geq 1$,

$$
\mathbf{P}\{\kappa(\mathbf{F}) \geq k+1\} \leq \sum_{i \geq k} \frac{1}{i !} \leq \frac{2}{k !} .
$$

Combining the latter equation with (14) yields that

$$
1 \leq(1+\varepsilon)^{j} e^{\frac{1}{2}} \mathbf{P}\left\{\mathbf{F} \in \mathcal{F}_{n, 1}\right\}+\alpha / 2,
$$

SO

$$
\mathbf{P}\left\{\mathbf{F} \in \mathcal{F}_{n, 1}\right\} \geq \frac{1-\alpha / 2}{(1+\varepsilon)^{j} e^{\frac{1}{2}}} \geq \frac{1-\alpha}{e^{\frac{1}{2}}}
$$


As $\alpha>0$ was arbitrary, (15) implies that $\mathbf{P}\{\mathbf{F}$ is connected $\} \geq e^{-\frac{1}{2}+o(1)}$ which combined with Lemma 4 proves Claim 3 .

A simpler version of the above argument lets us deduce directly from inequality (5) in Lemma 7 the non-asymptotic result, that $\mathbf{P}\{\mathbf{F}$ is connected $\}>e^{-n / W}$ for all positive integers $W$ and all weight vectors $\vec{w}=\left(w_{1}, \ldots, w_{n}\right)$ with $\sum_{j=1}^{n} w_{j}=W$.

\section{Proof of Lemma 5}

As already noted, Lemma 5 follows immediately from Lemma 7 in the special case when $n \leq \frac{1}{2} W$ : here we will prove the full result. Let $W$ be a positive integer and consider any positive integer weight vector $\vec{w}=\left(w_{1}, \ldots, w_{n}\right)$ with $\sum_{i} w_{i}=W$. We may assume that $n \geq 2$. Given a tree $T$ with vertex set $[n]:=\{1, \ldots, n\}$ and an edge $e \in T$, we denote by $s(T, e)$ the smaller weight component of $T-e$, or the component of $T$ containing vertex 1 if the components have equal weights. We call the components of $T-e$ pendant subtrees of $T$. For $i=1, \ldots,\lfloor W / 2\rfloor$, denote by $c(T, i)$ the quantity $|\{e \in T: w(s(T, e))=i\}|$.

Recall that $K=\sum_{F \in \mathcal{F}_{n}} \operatorname{mass}(F)$, and let $K^{\prime}=\sum_{T \in \mathcal{F}_{n, 1}} \operatorname{mass}(T)=K \cdot \mathbf{P}\left\{\mathbf{F} \in \mathcal{F}_{n, 1}\right\}$. Let $\mathbf{T}$ be a random tree with vertex set $\{1, \ldots, n\}$ and such that

$$
\mathbf{P}\{\mathbf{T}=T\}=\frac{\operatorname{mass}(T)}{K^{\prime}} .
$$

Our proof of Lemma 5 starts with the identity in Lemma 10 below, which expresses the ratio of $\mathbf{P}\left\{\mathbf{F} \in \mathcal{F}_{n, 2}\right\}$ to $\mathbf{P}\left\{\mathbf{F} \in \mathcal{F}_{n, 1}\right\}$ as a weighted sum of the values $\mathbf{E} c(\mathbf{T}, i)$. We then see that we can generate the random tree $\mathbf{T}$ in a natural way using Prüfer codes see Lemma 11. This lets us obtain a good upper bound on the probability that $\mathbf{T}$ has a pendant subtree with a given set of vertices (inequality (20) in Lemma 13); and using (20) we can upper bound the weighted sum mentioned above, and thus complete the proof.

Lemma 10.

$$
\mathbf{P}\left\{\mathbf{F} \in \mathcal{F}_{n, 2}\right\}=\mathbf{P}\left\{\mathbf{F} \in \mathcal{F}_{n, 1}\right\} \cdot \sum_{i=1}^{\lfloor W / 2\rfloor} \frac{\mathbf{E} c(\mathbf{T}, i)}{i(W-i)} .
$$

Proof. By Lemma 6 and the definition of the flow $\varphi$ given in (3),

$$
\begin{aligned}
K \cdot \mathbf{P}\left\{\mathbf{F} \in \mathcal{F}_{n, 2}\right\} & =\sum_{F^{\prime} \in \mathcal{F}_{n, 1}} \sum_{F \in \mathcal{F}_{n, 2}} \varphi\left(F^{\prime}, F\right) \\
& =\sum_{T \in \mathcal{F}_{n, 1}} \operatorname{mass}(T) \cdot \sum_{e \in T} \frac{1}{s(T, e)(W-s(T, e))} \\
& =\sum_{i=1}^{\lfloor W / 2\rfloor} \frac{1}{i(W-i)} \sum_{T \in \mathcal{F}_{n, 1}} \operatorname{mass}(T) \cdot c(T, i) .
\end{aligned}
$$

Also, for each $i=1, \ldots,\lfloor W / 2\rfloor$,

$$
\begin{aligned}
\sum_{T \in \mathcal{F}_{n, 1}} \operatorname{mass}(T) \cdot c(T, i) & =K \cdot \sum_{T \in \mathcal{F}_{n, 1}} \mathbf{P}\{\mathbf{T}=T\} \cdot \mathbf{P}\left\{\mathbf{F} \in \mathcal{F}_{n, 1}\right\} \cdot c(T, i) \\
& =K \cdot \mathbf{P}\left\{\mathbf{F} \in \mathcal{F}_{n, 1}\right\} \cdot \mathbf{E} c(\mathbf{T}, i) .
\end{aligned}
$$

Combining these results proves the lemma.

Lemma 10 allows us to understand the ratio between $\mathbf{P}\left\{\mathbf{F} \in \mathcal{F}_{n, 2}\right\}$ and $\mathbf{P}\left\{\mathbf{F} \in \mathcal{F}_{n, 1}\right\}$ by studying the values $\mathbf{E} c(\mathbf{T}, i)$ for $1 \leq i \leq\lfloor W / 2\rfloor$. Observe that, for any $k_{0}>0$, for $W \geq 2 k_{0}$,

$$
\sum_{k=k_{0}}^{\lfloor W / 2\rfloor} \frac{\mathbf{E} c(\mathbf{T}, k)}{k(W-k)} \leq \frac{n-1}{k_{0}\left(W-k_{0}\right)} \leq \frac{2}{k_{0}} .
$$


Thus it sufficies to show that for any $\varepsilon>0$, for $W$ sufficiently large we have

$$
\sum_{k=1}^{k_{0}} \frac{\mathbf{E} c(\mathbf{T}, k)}{k(W-k)} \leq(1+\varepsilon) \cdot \frac{1}{2} \text {. }
$$

Consider a sequence of iid random variables $Z_{1}, Z_{2}, \ldots, Z_{n-2}$ with $\mathbf{P}\left(Z_{1}=i\right)=\frac{w_{i}}{W}$ for $i=1, \ldots, n$, and let $\mathbf{Z}=\left(Z_{1}, \ldots, Z_{n-2}\right)$. Given $\mathbf{z}=\left(z_{1}, \ldots, z_{n-2}\right) \in[n]^{n-2}$ let $n_{i}(\mathbf{z})=\left|\left\{j: z_{j}=i\right\}\right|$ for each $i=1, \ldots, n$. We remind the reader that the Prüfer code construction (see for example the book by West [20]) gives a bijection $\phi:[n]^{n-2} \rightarrow \mathcal{F}_{n, 1}$ such that if $\phi(\mathbf{z})=T$ then $d_{T}(i)=n_{i}(\mathbf{z})+1$ for each $i$. (Recall that $\sum_{j} d_{T}(j)-1=n-2$ for any tree $T$ on $[n]$.)

Lemma 11. The random variables $\phi(\mathbf{Z})$ and $\mathbf{T}$ are identically distributed.

Proof. Fix any tree $T_{0}$ on $[n]$. There is exactly one sequence $\mathbf{z} \in[n]^{n-2}$ with $\phi(\mathbf{z})=T$, and this sequence must contain exactly $d_{T_{0}}(j)-1$ co-ordinates $j$ for each $j \in[n]$. We thus have

$$
\mathbf{P}\left(\phi(\mathbf{Z})=T_{0}\right)=\mathbf{P}(\mathbf{Z}=\mathbf{z})=\prod_{j=1}^{n}\left(\frac{w_{j}}{W}\right)^{d_{T_{0}}(j)-1}=\frac{\operatorname{mass}\left(T_{0}\right)}{\left(\prod_{j} w_{j}\right) \cdot W^{n-2}} .
$$

We must then have $K^{\prime}=\left(\prod_{j} w_{j}\right) \cdot W^{n-2}$ and, comparing with (16), the result follows.

Definition 12. Given $I \subseteq\{1, \ldots, n\}$, let $P_{I}$ be the event that $\mathbf{T}$ contains a pendant subtree $T$ with $V(T)=I$, and write $w(I)=\sum_{i \in I} w_{i}$.

Lemma 13. For each $I \subseteq[n]$,

$$
\mathbf{P}\left(P_{I}\right)=\left(\frac{w(I)}{W}\right)^{|I|-1}\left(1-\frac{w(I)}{W}\right)^{n-|I|-1} ;
$$

and so, for any $\delta>0$ and $w_{0}>0$, there is a $W_{0}$ such that for each $W \geq W_{0}$ and each $I \subseteq[n]$ with $w(I) \leq w_{0}$

$$
\mathbf{P}\left(P_{I}\right) \leq(1+\delta)\left(\frac{w(I)}{W}\right)^{|I|-1} e^{-\frac{n w(I)}{W}} .
$$

Proof. Let $I=\{1, \ldots, i\} \subseteq[n]$. According to the Prufer bijection, $I$ is the set of vertices in a pendant subtree of $\phi(\mathbf{z})$ where $\mathbf{z}=\left(z_{1}, \ldots, z_{n-2}\right)$, if and only if each of $z_{1}, \ldots, z_{i-1}$ is in $I$ and none of $z_{i}, \ldots, z_{n-2}$ is in $I$. Hence the probability that $I$ is the set of vertices in a pendant subtree of $\phi(\mathbf{Z})$ equals the right side of (19). This probability depends on the weights of the vertices in $I$ but does not depends on their labels, so (19) must hold for each set $I \subseteq[n]$. Further, the inequality $1-x \leq e^{-x}$ gives

$$
\left(1-\frac{w(I)}{W}\right)^{n-|I|-1} \leq e^{-\frac{n w(I)}{W}} \cdot e^{\frac{w(I)(|I|+1)}{W}},
$$

and so (19) yields (201).

Now a little manipulation of generating functions will allow us to use (20) to upper bound the sum on the right side of (17), and thus complete the proof.

Let $F(y)=\sum_{i=1}^{n} y^{w_{i}}$ and let $G(y)=\frac{1}{n} F(y)$. Next, let $X$ be uniformly distributed on $[n]$, and let $Y=w_{X}$, so that $Y$ has probability generating function $G(y)$. Let $Y_{1}, Y_{2}, \ldots$ be independent, each distributed like $Y$, and for $i \geq 1$ let $Z_{i}=Y_{1}+\cdots+Y_{i}$. Then for each positive integer $k$

$$
\left[y^{k}\right] G(y)^{i}=\mathbf{P}\left(Z_{i}=k\right):=p_{i}(k) .
$$


Thus

$$
\begin{aligned}
\sum_{I \subseteq[n], w(I)=k} x^{|I|} & =\left[y^{k}\right] \sum_{I \subseteq[n]} x^{|I|} y^{w(I)}=\left[y^{k}\right] \sum_{i \geq 1} x^{i} \sum_{I \subseteq[n],|I|=i} y^{w(I)} \\
& =\left[y^{k}\right] \sum_{i \geq 1} \frac{x^{i}}{i !} \sum_{x_{1}, \ldots, x_{i} \in[n] \text { distinct }} y^{\sum_{j} w_{x_{j}}} \\
& \leq\left[y^{k}\right] \sum_{i \geq 1} \frac{x^{i}}{i !} \sum_{x_{1}, \ldots, x_{i} \in[n]} y^{\sum_{j} w_{x_{j}}}=\left[y^{k}\right] \sum_{i \geq 1} \frac{x^{i}}{i !} F(y)^{i} \\
& =\sum_{i \geq 1} \frac{(n x)^{i}}{i !}\left[y^{k}\right] G(y)^{i}=\sum_{i \geq 1} \frac{(n x)^{i}}{i !} p_{i}(k) .
\end{aligned}
$$

(Since $p_{i}(k)=0$ for $i>k$ we could replace $i \geq 1$ in the sum above by $1 \leq i \leq k$.)

We will use this result with $x=\frac{k}{W}$. Let $\alpha=\frac{n}{W}$, so that $n x=\alpha k$. By the last inequality and (20) in Lemma 13, for each $\delta>0$ and each $k$, for $W$ sufficiently large (both for (20) and so that $\left.\left(1-\frac{k}{W}\right)^{-1} \leq(1+\delta)\right)$ we have

$$
\begin{aligned}
\frac{\mathbf{E} c(\mathbf{T}, k)}{k(W-k)} & =\frac{1}{k(W-k)} \sum_{I \subseteq[n], w(I)=k} \mathbf{P}\left(P_{I}\right) \\
& \leq(1+\delta)\left(1-\frac{k}{W}\right)^{-1} k^{-2} e^{-\frac{n k}{W}} \sum_{I \subseteq[n], w(I)=k}\left(\frac{k}{W}\right)^{|I|} \\
& \leq(1+\delta)^{2} k^{-2} e^{-\alpha k} \sum_{i \geq 1} \frac{(\alpha k)^{i}}{i !} p_{i}(k) .
\end{aligned}
$$

Now fix $\varepsilon>0$ and $k_{0}>0$. By the preceding inequality, for $W$ sufficiently large, for all $k \leq k_{0}$ we have

$$
(1+\varepsilon)^{-1} \cdot \frac{\mathbf{E} c(\mathbf{T}, k)}{k(W-k)} \leq k^{-2} e^{-\alpha k} \sum_{i \geq 1} \frac{(\alpha k)^{i}}{i !} p_{i}(k),
$$

and thus

$$
\begin{aligned}
(1+\varepsilon)^{-1} \cdot \sum_{k=1}^{k_{0}} \frac{\mathbf{E} c(\mathbf{T}, k)}{k(W-k)} & \leq \sum_{k=1}^{k_{0}} k^{-2} e^{-\alpha k} \sum_{i \geq 1} \frac{(\alpha k)^{i}}{i !} p_{i}(k) \\
& =\sum_{1 \leq i \leq k_{0}} \frac{\alpha^{i}}{i !} \sum_{k=i}^{k_{0}} k^{i-2} e^{-\alpha k} p_{i}(k) \\
& \leq \sum_{i \geq 1} \frac{\alpha^{i}}{i !} i^{-2} \sum_{k \geq i} k^{i} e^{-\alpha k} p_{i}(k) \\
& =\sum_{i \geq 1} \frac{\alpha^{i}}{i !} i^{-2} \mathbf{E}\left[Z_{i}^{i} e^{-\alpha Z_{i}}\right] .
\end{aligned}
$$

For $i=1,2, \ldots$ let $f_{i}(x)=x^{i} e^{-\alpha x}$ for $x>0$. Then $f^{\prime}(x)=x^{i-1} e^{-\alpha x}(i-\alpha x)$. Thus $f_{i}(x)$ takes its maximum value at $x=i / \alpha$, and its maximum value is $\left(\frac{i}{\alpha e}\right)^{i}$. Hence for $W$ 
sufficiently large

$$
\begin{aligned}
(1+\varepsilon)^{-1} \cdot \sum_{k=1}^{k_{0}} \frac{\mathbf{E} c(\mathbf{T}, k)}{k(W-k)} & \leq \sum_{i \geq 1} \frac{\alpha^{i}}{i !} i^{-2} \mathbf{E}\left[f_{i}\left(Z_{i}\right)\right] \\
& \leq \sum_{i \geq 1} \frac{\alpha^{i}}{i !} i^{-2}\left(\frac{i}{\alpha e}\right)^{i}=\sum_{i \geq 1} \frac{i^{i-2}}{i ! e^{i}}=\frac{1}{2} .
\end{aligned}
$$

The final equality above can be found, for example, in [7, page 109]. We have now established (18); this completes the proof of Lemma 5, and thus of Theorem 1 .

\section{CONClUding REMARKS}

We have proved the conjecture from McDiarmid, Steger and Welsh [17 that the class of forests is asymptotically the worst possible example of a bridge-addable graph class, from the point of view of connectivity, but only in the special case when the graph class is bridgealterable, and so also when the class is monotone as well as bridge-addable. Recently, a substantial amount of work has gone into counting the number of random graphs in a variety of graph classes that are bridge-addable; in some cases, this has also led to precise estimates on the probability of connectedness. Giménez and Noy [13] have shown that for a uniformly random planar graph, the probability of connectedness is approximately 0.963253 (correct to 6 decimal places, as are all the figures in this paragraph). The same results hold for the class of graphs embeddable on any fixed surface, Bender and Gao [3], Chapuy, Fusy, Giménez, Mohar and Noy [9]. Similarly, Bodirsky, Giménez, Kang and Noy [5, 6] have shown that for series-parallel graphs and outerplanar graphs, the probabilities of connectedness are approximately 0.889038 and 0.862082 , respectively; and Gerke, Giménez, Noy and Weißl [11] have shown that for random $K_{3,3}$-minor-free graphs, this probability is approximately 0.963262 . For further related results see [4, 10, 12, 14]. All these results are for monotone graph classes.

The original conjecture from [17] still is open. We venture the following, stronger conjecture.

Conjecture 1. For any $n$ and any non-empty bridge-addable set $\mathcal{A}$ of graphs on $\{1, \ldots, n\}$, if $\mathbf{G}$ is a uniformly random element of $\mathcal{A}$ and $\mathbf{F}$ is a uniformly random element of $\mathcal{F}_{n}$, then

$$
\mathbf{P}\{\mathbf{G} \text { is connected }\} \geq \mathbf{P}\{\mathbf{F} \text { is connected }\} .
$$

This conjecture would of course yield the original conjecture.

\section{REFERENCES}

[1] Balister, P., Bollobás, B. and Gerke, S. (2008) Connectivity of addable graph classes. J. Combin. Theory B 98577 - 584.

[2] Balister. P., Bollobás, B. and Gerke, S. (2010) Connectivity of random addable graphs. Proc. ICDM 2008, No 13, 2010, 127 - 134.

[3] Bender E. and Gao, Z. (2011) Asymptotic enumeration of labelled graphs by genus. Electronic J. Combin. 18 P13.

[4] Bernardi, O., Noy M. and D. Welsh, D. (2010) Growth constants of minor-closed classes of graphs. J. Combin. Theory B $100468-484$.

[5] Bodirsky, M., Giménez, O., Kang, M. and Noy, M. (2005) On the number of series parallel and outerplanar graphs. In Proceedings of the European Conference on Combinatorics, Graph Theory, and Applications (EuroComb '05), DMTCS Proceedings Series, pages 383-388.

[6] Bodirsky, M., Giménez, O., Kang, M. and Noy, M. (2007) Enumeration and limit laws for series-parallel graphs. European Journal of Combinatorics $282091-2105$. 
[7] Bollobás, B. (2001) Random Graphs. Cambridge Studies in Advanced Mathematics. Cambridge University Press, second edition.

[8] Cayley, A. (1889) A theorem on trees. Quart. J. Math. 23 376-378.

[9] Chapuy, G., Fusy, E., Giménez, O., Mohar, B. and Noy, M. (2011) Asymptotic enumeration and limit laws for graphs of fixed genus. J. Combin. Theory A 118748 777.

[10] Fountoulakis, N. and Panagioutou, K. (2011) 3-connected cores in random planar graphs. Combin. Probab. Comput $20381-412$.

[11] Gerke, S., Giménez, O., Noy, M. and Weißl, A. (2008) The number of graphs not containing $K_{3,3}$ as a minor. Electronic J. Combin. 15 R114.

[12] Giménez, O., Noy, M. and Rué, J.J. (2007) Graph classes with given 3-connected components: asymptotic counting and critical phenomena. Electronic Notes in Discrete Mathematics, 29:521-529.

[13] Giménez, O. and Noy, M. (2009) Asymptotic enumeration and limit laws of planar graphs. J. Amer. Math. Soc. 22 309-329.

[14] Giménez, O. and Noy, M. (2009) Counting planar graphs and related families of graphs. In Surveys in Combinatorics 2009, 169 - 329, Cambridge University Press, Cambridge.

[15] Kang, M. and Panagiotou, K. (2011) On the connectivity of random graphs from addable classes.

[16] McDiarmid, C., Steger, A. and Welsh, D. (2005) Random planar graphs. J. Combin. Theory B 93 187-206.

[17] McDiarmid, C., Steger, A. and Welsh, D. (2006) Random graphs from planar and other addable classes. In M. Klazar, J. Kratochvil, M. Loebl, J. Matousek, R. Thomas, and P. Valtr, editors, Topics in Discrete Mathematics, dedicated to Jarik Nesetril on the Occasion of his 60th birthday, volume 26 of Algorithms and Combinatorics, pages 231-246, Springer-Verlag.

[18] Moon, J.W. (1970) Counting labelled trees. Canadian Mathematical Monographs, Number 1.

[19] Rényi, A. (1959) Some remarks on the theory of trees. Publ. Math. Inst. Hungarian Acad. Sci. 4 73-85.

[20] West, D.B. (2001) Introduction to Graph Theory. Second edition, Prentice Hall.

Department of Mathematics and Statistics, McGill University, 805 Sherbrooke Street

West, Montréal, Québec, H3A 2K6, Canada

E-mail address: louigi@math.mcgill.ca

Department of Statistics, 1 South Parks Road, Oxford, OX1 3TG, UK

E-mail address: cmcd@stats.ox.ac.uk

School of Computer Science, McGill University, 3480 University Street, Montreal, QueBEC, H3A 2A7, CANADA

E-mail address: breed@cs.mcgill.ca 